\title{
Facial nerve motor evoked potentials during skull base surgery to monitor facial nerve function using the threshold-level method
}

\author{
Johannes Sarnthein, Ph.D., ${ }^{1,3}$ Nader Hejrati, Cand.Med., ${ }^{1}$ Marian C. Neidert, M.D., 1 \\ Alexander M. Huber, M.D., ${ }^{2}$ and Niklaus Krayenbühl, M.D. ${ }^{1}$ \\ Kliniken für ${ }^{1}$ Neurochirurgie und ${ }^{2}$ Ohren-, Nasen-, Hals-, und Gesichtschirurgie, UniversitätsSpital Zürich; \\ and ${ }^{3}$ Center for Integrative Human Physiology, Universität Zürich, Switzerland
}

Object. During surgeries that put the facial nerve at risk for injury, its function can be continuously monitored by transcranial facial nerve motor evoked potentials (FNMEPs) in facial nerve target muscles. Despite their advantages, FNMEPs are not yet widely used. While most authors use a 50\% reduction in FNMEP response amplitudes as a warning criterion, in this paper the authors' approach was to keep the response amplitude constant by increasing the stimulation intensity and to establish a warning criterion based on the "threshold-level" method.

Methods. The authors included 34 consecutive procedures involving 33 adult patients (median age 47 years) in whom FNMEPs were monitored. A threshold increase greater than $20 \mathrm{~mA}$ for eliciting FNMEPs in the most reliable facial nerve target muscle was considered a prediction of reduced postoperative facial nerve function, and subsequently a warning was issued to the surgeon. Preoperative and early postoperative function was documented using the House-Brackmann grading system.

Results. Monitoring of FNMEPs was feasible in all 34 surgeries in at least one facial nerve target muscle. The mentalis muscle yielded the best results. The House-Brackmann grade deteriorated in 17 (50\%) of 34 cases. The warning criterion was reached in $18(53 \%)$ of 34 cases, which predicted an $83 \%$ risk of House-Brackmann grade deterioration. Sensitivity amounted to $88 \%$ (CI 64\%-99\%) and specificity to $82 \%$ (CI 57\%-96\%). Deterioration of FNMEPs and a worse House-Brackmann grade showed a high degree of association $(\mathrm{p}<0.001)$. The impact of FNMEP monitoring on surgical strategy is exemplified in an illustrative case.

Conclusions. In surgeries that put the facial nerve at risk, the intraoperative increase in FNMEP stimulation threshold was closely correlated to postoperative facial nerve dysfunction. Monitoring of FNMEPs is a valid indicator of facial nerve function in skull base surgery. It should be used as an adjunct to direct electrical facial nerve stimulation and continuous electromyographic monitoring of facial nerve target muscles.

(http://thejns.org/doi/abs/10.3171/2012.12.FOCUS12386)

\section{KeY Words • facial nerve • motor evoked potential • threshold level • House-Brackmann grade}

ICRONEUROSURGERY of the skull base, especially of the CPA, still carries a significant risk of impairing facial nerve function. Among the techni-

\footnotetext{
Abbreviations used in this paper: $\mathrm{CPA}=$ cerebellopontine angle; DES = direct electrical stimulation; $\Delta \mathrm{I}_{\text {th }}=$ increase in FNMEP stimulation threshold with respect to the preoperative baseline; $\mathrm{EMG}=$ electromyography; FNMEP = facial nerve motor evoked potential; IONM = intraoperative neuromonitoring; ISI = interstimulus interval; $\mathrm{NPV}=$ negative predictive value $; \mathrm{PPV}=$ positive predictive value; TES = transcranial electrical stimulation.
}

cal measures to preserve facial nerve function, IONM has become mandatory and is constantly being advanced. ${ }^{3}$ During surgery, IONM serves to communicate impending nerve damage to the surgeon and to predict the postoperative neurological state. As a standard method of IONM, DES of the facial nerve serves to elicit compound muscle potentials recorded from facial nerve target muscles. Direct electrical stimulation is used to identify the facial nerve in the surgical field, to map its course, and for intermittent testing of facial nerve function. As another standard method, free-running EMG of facial nerve tar- 


\section{J. Sarnthein et al.}

get muscles provides continuous feedback of facial nerve activity.

More recently, FNMEPs have been introduced. . $^{4,7}$ Facial nerve MEPs allow activation of the motor pathway proximal to the surgical field by TES of the motor cortex and recording of responses in facial nerve target muscles. With this technique, facial nerve function can be monitored continuously. Despite this advantage, FNMEP monitoring has not yet become a standard tool of IONM, possibly due to artifacts in the signals and uncertainties in the interpretation of the results. We have therefore tested several electrode types to record FNMEPs. Most authors use the decrease in FNMEP response amplitude as a warning criterion..$^{1-4,8,12}$ However, FNMEP responses have inherent variability and are hard to quantify. Also, high FNMEP stimulation intensity is needed to achieve maximal FNMEP response already at baseline so that subsequent response deterioration can be assessed. Inspired by promising findings in IONM of motor function during surgery at the level of the spine, ${ }^{5}$ the cortex,${ }^{17}$ the brainstem, ${ }^{16}$ and the facial nerve, ${ }^{9}$ we propose here to monitor the increase in stimulation threshold needed to elicit FNMEPs to communicate with the surgeon and to predict postoperative facial nerve function.

\section{Methods}

\section{Patient Selection}

We included all consecutive adult patients who underwent surgical procedures within the CPA between August 2011 and December 2012 in whom IONM of facial nerve function was performed. Collection of personal patient data and scientific workup was approved by the institutional ethics review board (Kantonale Ethikkommission). The selection criterion resulted in a series of 34 surgical procedures in 33 patients (21 women; median age 47 years, range $20-84$ years). Patient characteristics are listed in Table 1.

\section{Pathological Conditions}

Table 1 lists the patients' pathological conditions. Twenty-two patients underwent surgery for vestibular schwannoma, 4 for meningioma, 4 for trigeminal neuralgia, and 4 patients had other pathologies (posterior inferior cerebellar aneurysm, brainstem cavernoma, medulloblastoma, and tuberculoma).

\section{Neurological Assessment}

Facial nerve function was scored according to the House-Brackmann Grading System (Grades I-VI: Grade I, normal facial muscle function; Grade VI, total palsy). ${ }^{10}$ Preoperative scores and scores on the 1st postoperative day were taken from patient records.

\section{Anesthesia Management}

Following the standard protocol for neurosurgical interventions, anesthesia was induced with intravenous application of the sedative drug propofol $(4-8 \mathrm{mg} / \mathrm{kg} / \mathrm{min})$, the opioid analgesic remifentanil (1-2 $\mu \mathrm{g} / \mathrm{kg} / \mathrm{min})$, and the skeletal muscle relaxant atracurium $(0.5 \mathrm{mg} / \mathrm{kg})$. After intubation, atracurium was omitted because of its interference with electrophysiological monitoring and mapping.

\section{Neurophysiological Monitoring Technique}

Intraoperative neurophysiological monitoring was performed using the ISIS system (inomed). Free-running EMG was recorded from facial nerve target muscles. Direct electrical stimulation for precise localization of the facial nerve in the surgical field was initiated with $0.2 \mathrm{~mA}$ at a distance from the facial nerve and was reduced to a minimal current of $0.05 \mathrm{~mA}$ as long as the facial nerve was well identified. Transcranial electric stimulation for FNMEP monitoring was performed using a constant current stimulator with a maximal stimulator output of 220 $\mathrm{mA}$. Limb MEP and FNMEP responses were amplified and filtered (100-3000 Hz) before display.

\section{Sites for Recording of Muscle Activity}

Among facial nerve target muscles, we recorded responses from the orbicularis oculi, nasalis, orbicularis oris, and mentalis muscles. Depending on the requirements of the surgery, in some patients only a subset of facial nerve target muscles was recorded. Thenar muscles served as target muscles to monitor upper-extremity MEP responses.

\section{Electrodes for Recording of Muscle Activity}

Thenar muscle MEP responses were recorded with pairs of noninsulated straight needle electrodes placed under the skin (stainless steel, $0.4 \times 12-\mathrm{mm}$ Neuroline Twisted pair, Ambu), typically overlying the target muscle belly. Impedance was typically less than $5 \mathrm{k} \Omega$. These electrodes were also used for facial nerve target muscles.

In an attempt to optimize recording of muscle activity, we also tested 2 other electrode types. Compared with straight needles, hook needle electrodes (stainless steel, $0.35 \times 12 \mathrm{~mm}$, Spes Medica) intrude into the skin for a shorter depth. We hypothesized that the shorter depth would increase specificity for muscle activity while still being easy to handle. Impedance was typically less than $5 \mathrm{k} \Omega$. As a third electrode type, we tested wire electrodes (stainless steel, 0.11-mm diameter, recording area 0.002 $\mathrm{mm}^{2}$, Spes Medica) that record activity very specific to the target muscle since only a very small area is exposed from insulation, which results in high impedance (typically $>50 \mathrm{k} \Omega$ ).

\section{Transcranial Electrical Stimulation}

Transcranial electrical stimulation current was delivered through 2 Corkscrew electrodes (SGM) placed at electrode sites $\mathrm{C} 3 / \mathrm{C} 4$ versus $\mathrm{Cz}$. While the montage $\mathrm{C} 3$ versus $\mathrm{C} 4$ is optimal for low MEP stimulation thresholds for the upper extremities, ${ }^{18}$ we chose stimulation against $\mathrm{Cz}$ to reduce neck movements disturbing to the surgeon. A bite block was placed in the mouth to prevent bite injuries of the tongue resulting from motor stimulation of the jaw.

Transcranial electrical stimulation was performed by applying rectangular pulses. To reduce the stimulation artifact in recordings, the pulse shape was symmetrically biphasic with positive and negative deflections during the 
FNMEPs analyzed using the threshold-level method

TABLE 1: Characteristics in 33 patients undergoing 34 procedures during which FNMEPs were monitored*

\begin{tabular}{|c|c|c|c|c|c|c|c|c|}
\hline $\begin{array}{l}\text { Case } \\
\text { No. }\end{array}$ & $\begin{array}{l}\text { Age (yrs), } \\
\text { Sex }\end{array}$ & Pathology & $\begin{array}{c}\text { Preop HB } \\
\text { Grade }\end{array}$ & $\begin{array}{l}\text { Postop HB } \\
\text { Grade }\end{array}$ & $\begin{array}{c}\text { HB Grade } \\
\text { Deterioration }\end{array}$ & $\begin{array}{c}\text { Preop } \\
\text { FNMEP }(\mathrm{mA})\end{array}$ & $\begin{array}{c}\text { Postop } \\
\text { FNMEP }(\mathrm{mA})\end{array}$ & $\begin{array}{c}\text { FNMEP Increase } \\
>20 \mathrm{~mA}\end{array}$ \\
\hline 1 & $20, M$ & vestibular schwannoma & I & I & no & 110 & 110 & no \\
\hline 2 & $37, \mathrm{~F}$ & trigeminal neuralgia & I & I & no & 140 & 140 & no \\
\hline 3 & $54, \mathrm{M}$ & vestibular schwannoma & I & I & no & 75 & 80 & no \\
\hline 4 & $67, M$ & vestibular schwannoma & I & $\|$ & yes & 135 & 140 & no \\
\hline 5 & $56, F$ & vestibular schwannoma & 1 & I & no & 85 & 92 & no \\
\hline 6 & $36, M$ & vestibular schwannoma & I & I & no & 100 & 110 & no \\
\hline 7 & $70, \mathrm{~F}$ & vestibular schwannoma & $\|$ & $\|$ & no & 90 & 100 & no \\
\hline 8 & $46, F$ & trigeminal neuralgia & I & I & no & 60 & 70 & no \\
\hline 9 & $69, \mathrm{M}$ & vestibular schwannoma & I & I & no & 185 & 200 & no \\
\hline 10 & $64, F$ & $\begin{array}{l}\text { AVM w/ associated PICA } \\
\text { aneurysm }\end{array}$ & 1 & I & no & 75 & 90 & no \\
\hline 11 & $82, \mathrm{~F}$ & trigeminal neuralgia & I & I & no & 75 & 90 & no \\
\hline 12 & $46, F$ & meningioma & I & I & no & 45 & 60 & no \\
\hline 13 & $33, \mathrm{M}$ & meningioma & 1 & I & no & 80 & 95 & no \\
\hline 14 & $67, \mathrm{~F}$ & trigeminal neuralgia & I & I & no & 65 & 80 & no \\
\hline 15 & $43, \mathrm{~F}$ & vestibular schwannoma & I & III & yes & 55 & 75 & no \\
\hline 16 & $47, \mathrm{~F}$ & vestibular schwannoma & I & I & no & 60 & 80 & no \\
\hline 17 & $47, \mathrm{~F}$ & meningioma & I & ॥ & yes & 135 & 160 & yes \\
\hline 18 & $31, F$ & vestibular schwannoma & I & $\|$ & yes & 80 & 110 & yes \\
\hline 19 & $52, \mathrm{M}$ & meningioma & I & III & yes & 65 & 95 & yes \\
\hline 20 & $49, \mathrm{~F}$ & vestibular schwannoma & I & III & yes & 65 & 95 & yes \\
\hline 21 & $38, M$ & vestibular schwannoma & I & IV & yes & 60 & 90 & yes \\
\hline 22 & $48, F$ & vestibular schwannoma & I & ॥ & yes & 80 & 110 & yes \\
\hline 23 & $46, F$ & vestibular schwannoma & I & IV & yes & 80 & 110 & yes \\
\hline 24 & $58, \mathrm{~F}$ & vestibular schwannoma & I & $\|$ & yes & 50 & 85 & yes \\
\hline 25 & $21, F$ & vestibular schwannoma & I & $\|$ & yes & 100 & 140 & yes \\
\hline 26 & $72, \mathrm{~F}$ & vestibular schwannoma & I & V & yes & 40 & 85 & yes \\
\hline 27 & $43, M$ & vestibular schwannoma & I & $\|$ & yes & 65 & 110 & yes \\
\hline 28 & $46, F$ & vestibular schwannoma & I & III & yes & 100 & 150 & yes \\
\hline 29 & $30, F$ & tuberculoma & I & $\|$ & yes & 60 & 110 & yes \\
\hline 30 & $21, M$ & medulloblastoma & I & I & no & 80 & 140 & yes \\
\hline 31 & $84, \mathrm{~F}$ & vestibular schwannoma & 1 & 1 & no & 90 & 150 & yes \\
\hline 32 & $37, \mathrm{M}$ & brainstem cavernoma & VI & V & no & 80 & 150 & yes \\
\hline 33 & $40, M$ & vestibular schwannoma & I & III & yes & 90 & 160 & yes \\
\hline 34 & $58, \mathrm{M}$ & vestibular schwannoma & I & VI & yes & 60 & 150 & yes \\
\hline
\end{tabular}

* $\mathrm{AVM}=$ arteriovenous malformation; $\mathrm{HB}=$ House-Brackmann; $\mathrm{PICA}=$ posterior inferior cerebellar artery.

pulse width. Only the phase of anodal current was considered in the calculation of the effective charge that was delivered to the patient. The width of the anodal phase ranged between 0.2 and $0.5 \mathrm{msec}$ and was typically $0.4 \mathrm{msec}$.

Transcranial electrical stimulation pulses were applied in trains of 3-6 pulses for FNMEPs and MEPs. While an ISI of $4 \mathrm{msec}$ is optimal for limb MEPs, ${ }^{18}$ we used an ISI of $2 \mathrm{msec}$ to widen the interval between the TES artifact in the signal traces and the FNMEP response.

To distinguish between corticobulbar and peripheral stimulation, ${ }^{20}$ we added a control pulse either $20 \mathrm{msec}$ before or $40 \mathrm{msec}$ after the pulse train. Parameters were chosen so that for at least one facial nerve target muscle the TES pulse train elicited a response but the control pulse did not.

\section{Determination of MEP Stimulation Threshold}

The baseline FNMEP stimulation threshold was determined before skin incision. To obtain the baseline MEP threshold, we started by using a fixed pattern of stimulus intensity at $30 \mathrm{~mA}$, which increased by $5-\mathrm{mA}$ increments until one of the target muscles responded reliably to stimulation. At least 2 seconds elapsed from one stimulus train to another. An evoked FNMEP response as low as $20 \mu \mathrm{V}$ with appropriate response latency qualified as a reliable FNMEP response, ${ }^{6,16}$ although responses 
were typically more than $100 \mu \mathrm{V}$. The testing was repeated continuously and at short intervals when facial nerve function was assumed to be at risk.

\section{Electrophysiological Data Analysis}

Whenever reduced FNMEP amplitude responses were observed, technical failures were ruled out primarily, and anesthesia parameters were checked. Secondarily, the number of stimulating pulses and subsequently the FNMEP stimulation intensity were elevated with the aim of obtaining a constant FNMEP response amplitude. At a higher stimulus intensity, disturbing muscle reactions necessitated precise synchronization of FNMEPs with surgical requirements. Gradually progressive threshold elevations were attributed to anesthetic fade,${ }^{11}$ and the baseline FNMEP threshold was adjusted. ${ }^{6,16}$ Rapid threshold elevations were analyzed in the context of the surgical manipulations and were considered possibly pathological. Facial nerve MEPs were considered deteriorated, and the surgical team was notified whenever the FNMEP intensity threshold had to be elevated by more than 20 $\mathrm{mA}$. Data analysis focused on those facial nerve target muscles with the most salient FNMEP responses and in which corticobulbar - as opposed to peripheral-stimulation was most clearly evident.

\section{Statistical Analysis}

Statistical analyses were performed using custom scripts in Matlab R2010a (The MathWorks, Inc.). For ratios, the $95 \%$ confidence intervals were obtained on the basis of the binomial distribution. Distributions were compared using nonparametric testing. Statistical significance was established as $\mathrm{p}<0.05$.

The outcomes of FNMEP and neurological examinations were dichotomized for statistical treatment in contingency tables with the chi-square test. A contingency table contains the elements true positive (TP), true negative (TN), false positive (FP), and false negative (FN). Derivations of these are the sensitivity or true-positive rate, $\mathrm{TPR}=\mathrm{TP} /(\mathrm{TP}+\mathrm{FN})$; the false-positive rate, $\mathrm{FPR}$ $=\mathrm{FP} /(\mathrm{FP}+\mathrm{TN})$; the accuracy, $\mathrm{ACC}=(\mathrm{TP}+\mathrm{TN}) /(\mathrm{TP}$ $+\mathrm{TN}+\mathrm{FP}+\mathrm{FN})$; the specificity: 1 - FPR; the negative predictive value, $\mathrm{NPV}=\mathrm{TN} /(\mathrm{TN}+\mathrm{FN})$; and the positive predictive value, $\mathrm{PPV}=\mathrm{TP} /(\mathrm{TP}+\mathrm{FP})$.

\section{Results}

\section{Representative FNMEP Responses}

Figure 1 shows representative traces recorded from facial nerve target muscles in a patient (Case 26) at baseline. The control pulse was delivered $20 \mathrm{msec}$ before the train of 5 pulses. None of the muscles responded to the control pulse, which excludes peripheral stimulation for the chosen set of stimulation parameters. The train of 5 pulses elicits a response in all muscles. The response latency exceeds $20 \mathrm{msec}$ for thenar muscles. Facial nerve target muscles show a variety of response latencies, and the responses are polyphasic to a varying degree. The variability of responses may be related to the modification of facial nerve anatomy in the presence of the lesion.

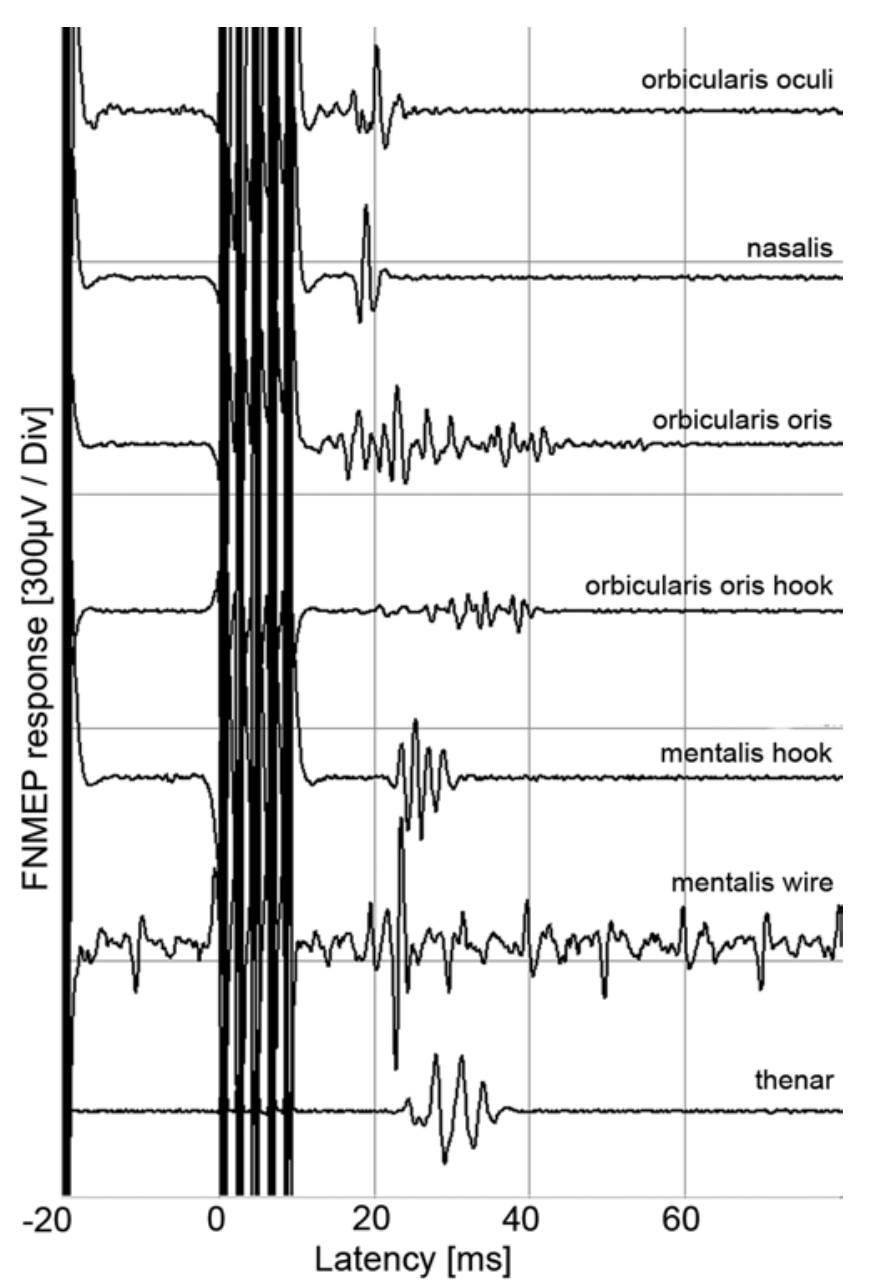

FIG. 1. Case 26. Representative FNMEP and MEP responses. The traces were recorded at baseline. The artifact of the TES control pulse is visible at $-20 \mathrm{msec}$, which does not elicit muscle activity. The TES train of 5 pulses elicits polyphasic responses at various latencies. Pulses were biphasic and the width of the anodal phase was $0.25 \mathrm{msec}$. Signals recorded from hook needle electrodes are labeled "hook," and the signal from the wire electrode is labeled "wire." All other signals were recorded from straight needle electrodes.

Impedances were less than $5 \mathrm{k} \Omega$ for straight and hook needles and around $100 \mathrm{k} \Omega$ for the wire electrode. The recording from the wire electrode stands out as it is contaminated by a $50-\mathrm{Hz}$ line hum. The low signal-to-noise ratio made it difficult to interpret the results of FNMEPs, the free-running EMG, and the localization of the facial nerve by DES in the surgical field. The wire electrode was therefore only used in a limited number of cases.

\section{Threshold Increase of FNMEPs Is Correlated With Postoperative House-Brackmann Grade}

Figure 2 shows $\Delta \mathrm{I}_{\mathrm{th}}$ (the increase in FNMEP stimulation threshold) as a function of the postoperative HouseBrackmann grade. Depicted is the value of $\Delta \mathrm{I}_{\text {th }}$ for the facial nerve target muscle that corresponded most reliably to FNMEPs. Under physiological considerations, stimulation intensity should be depicted as charge. From a practical point of view, the current delivered is readily available 
FNMEPs analyzed using the threshold-level method

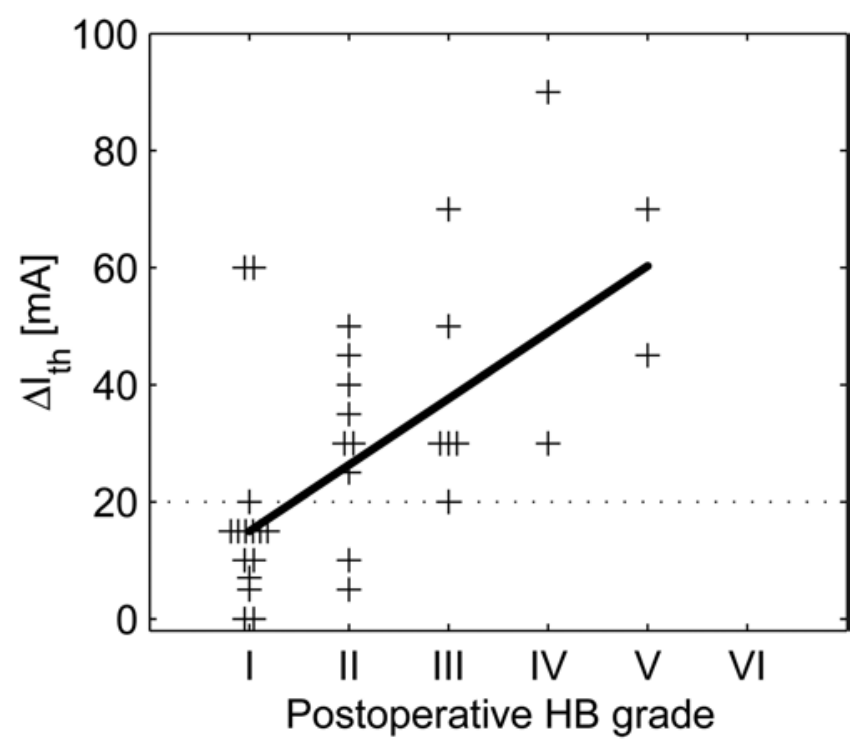

FIG. 2. The FNMEP threshold increase is correlated with postoperative House-Brackmann grade. Shown is $\Delta \mathrm{I}_{\mathrm{th}}$, the intraoperative increase in FNMEP threshold for the facial nerve target muscle that corresponded most reliably, as a function of postoperative House-Brackmann (HB) grade (Spearman correlation rho 0.62, $p<0.001$ ). The diagonal line represents a linear fit to the data. The dotted line at $20 \mathrm{~mA}$ indicates the threshold level at which a warning was issued to the surgical team. Warnings were issued in $18(53 \%)$ of 34 surgeries.

from our IONM device. We therefore depict the increase of stimulation current. The postoperative House-Brackmann grade and the increase in current needed to elicit FNMEPs was correlated with a rho of $0.62(\mathrm{p}<0.001$, Spearman).

\section{Intraoperative Warnings Are Associated With House-Brackmann Grade Deterioration}

Preoperative and early postoperative House-Brackmann grades for all cases are listed in Table 1. The HouseBrackmann grade deteriorated in 17 of 34 cases $(50 \%$, CI $32 \%-68 \%$ ). During the course of surgery, the threshold to elicit FNMEP responses increased by $\Delta \mathrm{I}_{\text {th }}$ greater than 20 $\mathrm{mA}$ in 18 of 34 cases (53\%, CI 35\%-70\%), and a warning was issued to the surgical team (Table 1).

Of all 34 cases, $\mathrm{TN}=14$ had no warning and no House-Brackmann deterioration, TP $=15$ had FNMEP warnings and House-Brackmann deterioration, FP = 3 had warnings but no new deficit, and $\mathrm{FN}=2$ had no warning but a new deficit. Thus, FNMEP deterioration to the warning criterion predicts an $83 \%$ risk of HouseBrackmann deterioration (PPV, CI 59\%-96\%). With 14 true negative cases of all 16 negative cases, the NPV was 88\% (CI 62\%-98\%). In 17 cases House-Brackmann deteriorated, and a warning had been issued in 15 of them (sensitivity 88\%, CI 64\%-100\%). In 17 cases the HouseBrackmann grade remained unchanged, and in 14 of these cases the FNMEPs remained stable (specificity $82 \%$, CI $57 \%-96 \%$ ). For all patients, the occurrence of deteriorated FNMEPs and deteriorated House-Brackmann grade showed a high degree of association $(p<$ 0.001 , chi-square test).

\section{Effects of Varying the Warning Criterion}

In Fig. 3 the receiver operating characteristic curve shows the predictive power of FNMEPs as the warning criterion was varied. The numbers denote the points on the curve where the warning criterion $\Delta \mathrm{I}_{\mathrm{th}}$ was at 20 and at $15 \mathrm{~mA}$. The area under the curve is 0.80 .

Concerning surgical outcome, the PPV is of particular importance. Figure 4 shows the derivations of the contingency table as a function of the warning criterion, that is, the increase in stimulation threshold $\Delta \mathrm{I}_{\mathrm{th}}$. With increasing threshold, a maximal accuracy in prediction is achieved for a $\Delta \mathrm{I}_{\mathrm{th}}$ of $20 \mathrm{~mA}$. With the warning criterion at $20 \mathrm{~mA}$, the fact of issuing a warning was associated with a PPV risk of $83 \%$ of postoperative deterioration of facial nerve function.

Stimulation Parameters, Facial Nerve Target Muscles, and Recording Electrode Types

The median stimulation threshold to elicit FNMEP responses at baseline was $75 \mathrm{~mA}$, which corresponds to a pulse with charge $26 \mu \mathrm{C}$. The threshold was higher than the MEP threshold to elicit responses at the thenar muscle (median $63 \mathrm{~mA}, 22 \mu \mathrm{C})$ in $25(83 \%)$ of 30 patients $(\mathrm{p}<$ 0.001 , sign test). Monitoring of FNMEPs was feasible in all 34 procedures in at least one facial nerve target muscle. Not all muscles were monitored in all patients.

We obtained reliable results in $15(50 \%)$ of 30 recordings at the orbicularis oculi muscle, in 6 (24\%) of 25 recordings at the nasalis muscle, in $33(69 \%)$ of 48 recordings at the orbicularis oris muscle, and in 48 (84\%) of 57 recordings at the mentalis muscle. The mentalis muscle yielded the best result among all facial nerve target muscles. The rates for different electrode types are presented in Table 2. Contrary to our initial expectation, straight needle electrodes produced the best results.

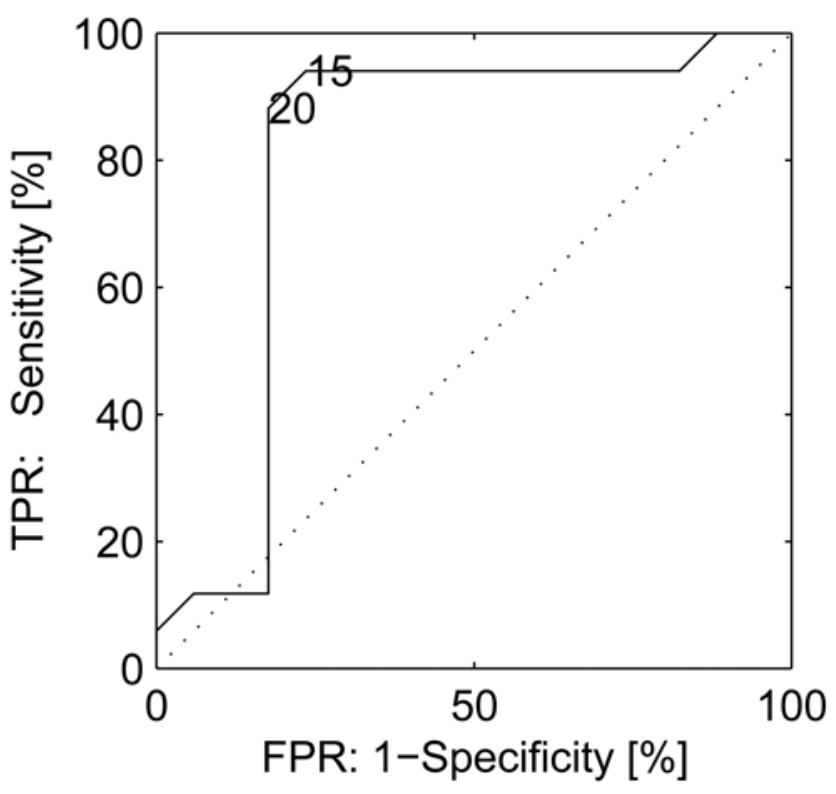

FIG. 3. Receiver operating characteristic curve. The sensitivity and specificity of FNMEP as the warning criterion is varied are shown. The numbers in the curve denote the points on the curve at which $\Delta \mathrm{l}_{\text {th }}$ was set to $20 \mathrm{~mA}$ and $15 \mathrm{~mA}$. The area under the curve is 0.80 . FPR $=$ falsepositive rate; TPR = true-positive rate. 

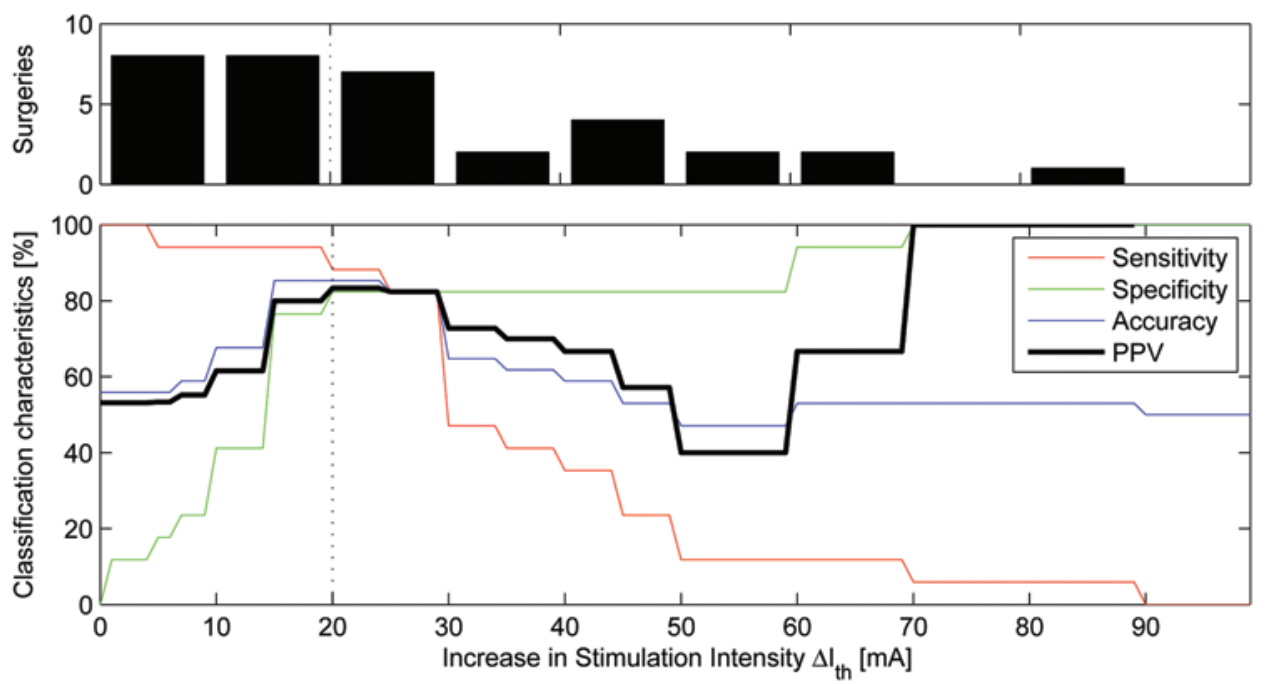

FIG. 4. Derivations of the contingency table as a function of the warning criterion. Upper: Distribution of cases in our group of surgeries. Lower: With increasing threshold $\Delta \mathrm{I}_{\text {th }}$, a maximal accuracy in prediction is achieved for $\Delta \mathrm{l}_{\mathrm{th}}$ at $20 \mathrm{~mA}$. Lowering $\Delta \mathrm{I}_{\text {th }}$ increases sensitivity at the expense of specificity.

\section{Illustrative Case}

\section{Case 21: Surgical Strategy Influenced by FNMEP} Recording

This 38-year-old man with House-Brackmann Grade I function presented with a vestibular schwannoma that was $30 \mathrm{~mm}$ in diameter. At a later stage of surgery, DES of the facial nerve proximal to the surgical field did not elicit muscle responses. Based solely on this finding, one could have assessed facial nerve function to be lost and chosen a surgical strategy of rapid radical tumor resection. However, FNMEPs could still be elicited (Fig. 5), albeit only in the mentalis muscle and at a highly elevated stimulation threshold of $90 \mathrm{~mA}$ (baseline $60 \mathrm{~mA}$ ). This led to the conclusion that the loss of DES response might have been due to anatomical changes caused by the tumor. Therefore, the surgeon opted for less radical tumor resection. Postoperative evaluation revealed only moderately severe facial nerve dysfunction (House-Brackmann Grade IV). In this case, the presence of FNMEP recordings-as opposed to DES alone-contributed to preservation of facial nerve function.

\section{Discussion}

\section{The "Threshold-Level" Method for FNMEP Interpretation}

Motor evoked potentials are a well-established component of IONM of corticospinal tract function. ${ }^{13}$ More recently, the indication for MEP monitoring has been extended to also monitor the function of the corticobulbar tract, and, in particular, facial nerve function. ${ }^{4,7}$ Most authors consider a reduction in FNMEP response amplitude of more than $50 \%$ as a warning criterion ${ }^{1-4,7,8,12}$ as widely accepted for MEPs in supratentorial surgery. ${ }^{15,19}$ The threshold-level method was first proposed for monitoring spinal cord function. ${ }^{5}$ There is one report of FNMEP threshold monitoring in the literature. ${ }^{9}$ We report here a series of surgeries in which FNMEPs were analyzed by the threshold-level method to monitor facial nerve function intraoperatively. With this approach we obtained a sensitivity of $88 \%$ and a specificity of $82 \%$, which is in the range of values reported by other authors., ${ }^{2,7,7}$ With the warning criterion at $20 \mathrm{~mA}$, an intraoperative warning was associated with a risk of PPV of $83 \%$ (CI 59\%$96 \%$ ) of postoperative deterioration of facial nerve function. A high NPV of $88 \%$ (CI 62\%-98\%) indicates that the absence of an intraoperative warning predicts good postoperative facial nerve function.

\section{Clinical Value of FNMEP Monitoring}

The clinical value of FNMEP monitoring is 2-fold. On one hand, DES proximal to the lesion may fail to produce responses in facial nerve target muscles, as shown in our case description (Fig. 5). Then the fact that FNMEPs can be elicited indicates that facial nerve function is not totally lost. Based on this information the surgeon refrained from aggressive tumor resection, and facial nerve function could be preserved.

On the other hand, an increase in stimulation threshold $\Delta \mathrm{I}_{\mathrm{th}}$ informs the surgeon about possible postoperative deterioration of facial nerve function if a warning criterion is reached. The criterion adopted in our study was motivated by values published for surgeries at the level of

TABLE 2: Comparison of EMG electrode types

\begin{tabular}{llc}
\hline \multicolumn{1}{c}{ Muscle } & Electrode Type & $\begin{array}{c}\text { No. of Successful Recordings/ } \\
\text { All Recordings (\%) }\end{array}$ \\
\hline orbicularis oculi & straight needle & $15 / 30(50)$ \\
nasalis & straight needle & $6 / 25(24)$ \\
orbicularis oris & straight needle & $22 / 31(71)$ \\
& hook needle & $11 / 17(65)$ \\
mentalis & straight needle & $23 / 25(92)$ \\
& hook needle & $22 / 27(81)$ \\
& wire & $3 / 5(60)$ \\
\hline
\end{tabular}




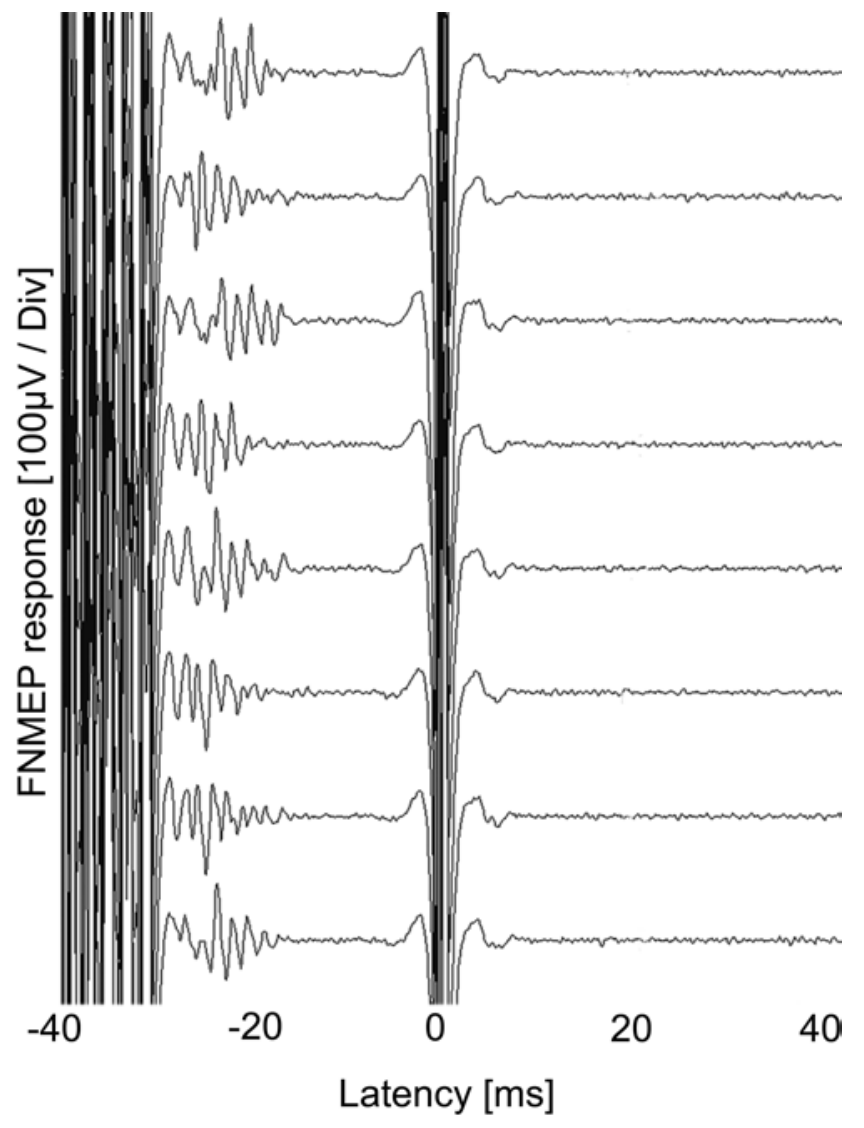

FIG. 5. Case 21. Persisting FNMEPs in the absence of facial nerve responses to direct facial nerve stimulation. The mentalis muscle FNMEPs at the end of surgery are shown, 1-minute interval between traces. The stimulation artifact of a TES train of 6 pulses is seen at -40 msec (biphasic pulses, anodal phase width $0.5 \mathrm{msec}$ ). The train elicits a polyphasic FNMEP response in the mentalis muscle. The control pulse at 0 msec does not elicit muscle activity.

the spine, ${ }^{5}$ the cortex,${ }^{17}$ and the brainstem. ${ }^{16}$ This criterion led to a warning issued in $18(53 \%)$ of 34 surgical interventions. However, one has to bear in mind that the 3 instances of warnings without ensuing House-Brackmann grade deterioration cannot strictly qualify as false-positive cases since action was taken intraoperatively with the aim to prevent paresis.

There is a tradeoff between sensitivity and specificity as shown by the receiver operating curve in Fig. 3. We found that the highest accuracy (85\% [CI 69\%-95\%]) for $\Delta \mathrm{I}_{\mathrm{th}}$ is $20 \mathrm{~mA}$, which was associated with a PPV of $83 \%$. This PPV may be considered high, since a warning predicts an $83 \%$ chance of reduced postoperative facial nerve function. With a lower $\Delta \mathrm{I}_{\text {th }}$ as a criterion, warnings are issued earlier and are associated with a lower chance of reduced postoperative facial nerve function. In general, more sensitive warning criteria have been shown to trigger surgical reevaluation earlier. ${ }^{6,14}$ If we reduce $\Delta \mathrm{I}_{\mathrm{th}}$ to 15 $\mathrm{mA}, \mathrm{PPV}$ is reduced to $80 \%$, which is only a small effect (Fig. 4). To achieve a substantial reduction to a PPV of $62 \%$, the warning criterion must be lowered to $10 \mathrm{~mA}$. This in turn reduces specificity to $41 \%$, which may jeopardize the credibility of FNMEP monitoring. Furthermore, most patients in our series showed only a mild deteriora- tion of facial nerve function when looking at changes in House-Brackmann grades. Based on long-term studies in the literature we expect an improvement of facial nerve function at later follow-up visits. ${ }^{1,8,12}$ Regarding changes of intraoperative surgical strategy, a simple algorithm based on FNMEPs cannot be defined. Considering conflicts between postoperative facial nerve function and completeness of tumor resection, surgical management decisions should also depend on individual patient factors such as tumor entity (malignant vs benign), patient age, and individual tolerance of facial nerve deficits.

For future surgeries we propose to not simply issue a warning to the surgeon, but to inform him or her in a more detailed way based on the findings of this study. The threshold increase $\Delta \mathrm{I}_{\mathrm{th}}$ is reported together with the PPV, that is, the associated risk for any deterioration of postoperative facial nerve function (Fig. 4). In addition, the degree of deterioration can be estimated from the amount of threshold increase (Fig. 2).

\section{Advantages of the Threshold-Level Method}

Warning criteria based on the FNMEP response amplitude have some drawbacks. First, there is inherent variability in the amplitude of the muscle responses; FNMEP responses are often polyphasic and extended over time so that they are difficult to quantify. Second, high FNMEP stimulation intensity is needed to achieve maximal FNMEP response already at baseline so that subsequent response deterioration can be assessed. High MEP stimulation intensity may cause movements such as neck twitches, which are disturbing to the surgical procedure.

As a different approach, the threshold-level method uses lower FNMEP stimulation intensity at onset. This approach aims to keep the response amplitude constant throughout surgery by adapting the FNMEP stimulation intensity. It has been suggested that in event of deterioration of corticospinal tract function, this method may provide a warning earlier. ${ }^{6}$

As a further simplification, we focused our analysis on the facial nerve target muscle that gave the most reliable response (mentalis muscle in $87 \%$ of recordings). In our opinion this approach is feasible since fibers of all muscle branches are running in a close topographic relation within the compact facial nerve course in the CPA. In contrast to procedures that involve the peripheral branches of the facial nerve, it seems unlikely that facial nerve injuries during CPA procedures involve only a subset of facial nerve muscles. As expected from studies that show a high correlation between different branches of the facial nerve, ${ }^{2,8}$ the analysis of the best responding muscle was representative and useful for predicting postoperative facial nerve function as defined by the House-Brackmann grading system.

\section{Distinguishing Between Corticobulbar and Peripheral Stimulation}

The main value of FNMEPs is to activate the corticobulbar tract proximal to the surgical field and to record responses of facial nerve target muscles to monitor the 
function of the tract (Fig. 6). Because of the proximity between the stimulating electrodes and the facial nerve target muscles, peripheral stimulation may also occur. Several criteria have been proposed to distinguish between corticobulbar and peripheral stimulation. ${ }^{2-4,7-9,12}$

Ideally, a corticobulbar FNMEP response should occur after a latency of about $10 \mathrm{msec}$ and it should be polyphasic. Furthermore, it has been claimed that a corticobulbar response requires stimulation by a train of several pulses, and response to a single pulse is due to peripheral stimulation. ${ }^{7,20}$ In the double train approach we have therefore added a train consisting of a single control pulse to the train of 3-6 pulses, which was intended to evoke FNMEP responses. Only if a response to the control pulse was absent, was the response to the train qualified to be a corticobulbar FNMEP.

\section{Conclusions}

In surgeries that put the facial nerve at risk, the in-

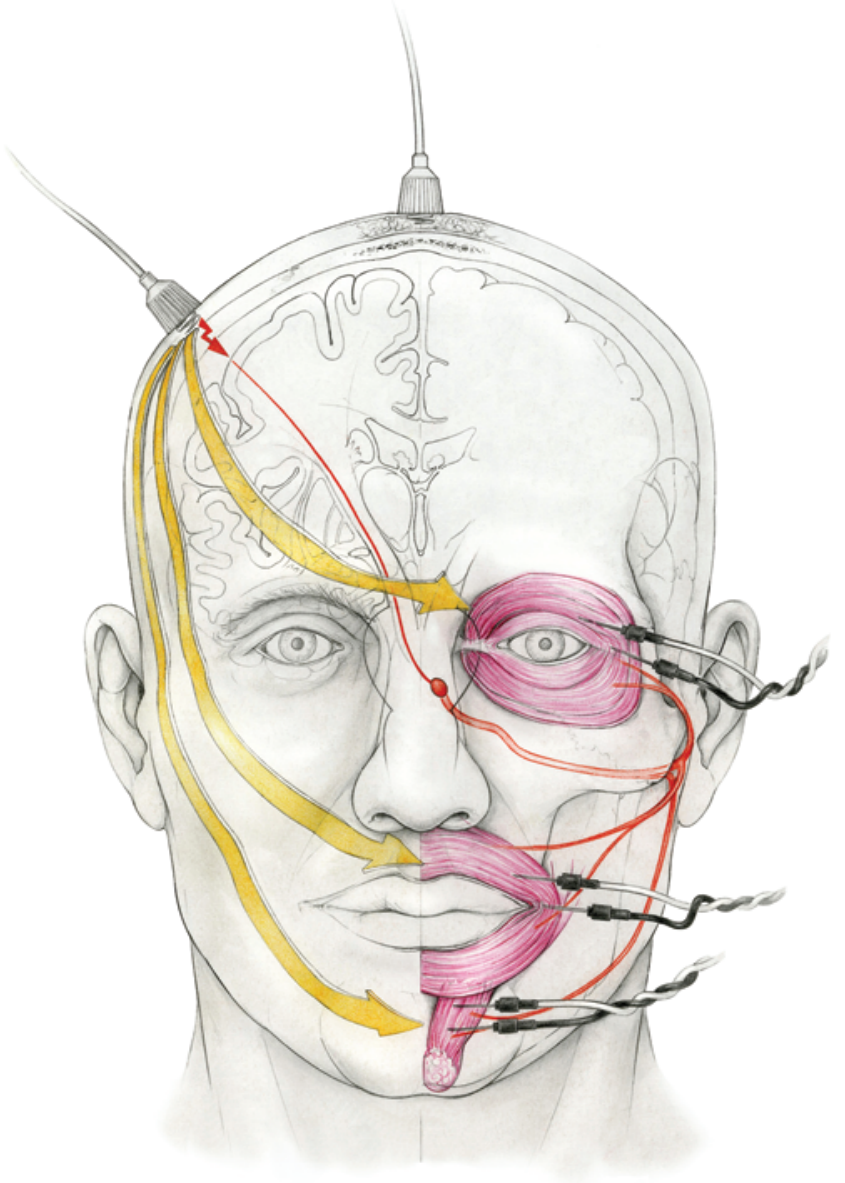

FIG. 6. Illustration showing the activation of the corticobulbar tract versus peripheral stimulation of facial nerve target muscles. During activation of the corticobulbar tract, anodal stimulation of the motor cortex (red arrow) elicits activation of lower motor neurons in the facial nerve nucleus of the brainstem, from which facial nerve target muscles are activated. As a confounder, peripheral stimulation (yellow arrows) may also activate facial nerve target muscles, albeit at shorter latencies and already in response to single stimulation pulses. Printed with permission from J. Sarnthein. traoperative increase in stimulation threshold was closely correlated to postoperative facial nerve dysfunction. A threshold increase greater than $20 \mathrm{~mA}$ predicted an $83 \%$ risk for deterioration in House-Brackmann grade. Monitoring of FNMEPs is a valid indicator of postoperative facial nerve function in skull base surgery. It should be used as an adjunct to direct electrical facial nerve stimulation and continuous EMG monitoring of facial nerve target muscles.

\section{Disclosure}

The authors report no conflict of interest concerning the materials or methods used in this study or the findings specified in this paper. J. Sarnthein has received consulting fees for serving as a speaker for inomed Co., Germany.

Author contributions to the study and manuscript preparation include the following. Conception and design: Sarnthein. Acquisition of data: Sarnthein, Hejrati, Huber, Krayenbühl. Analysis and interpretation of data: Sarnthein, Hejrati, Neidert. Drafting the article: Sarnthein, Neidert, Hejrati. Critically revising the article: Sarnthein, Neidert. Reviewed submitted version of manuscript: all authors. Approved the final version of the manuscript on behalf of all authors: Sarnthein. Statistical analysis: Sarnthein.

\section{Acknowledgments}

The authors thank E. Ciessynna for help in electrode placement and FNMEP recording. They also thank Peter Roth for providing the artwork shown in Figure 6.

\section{References}

1. Acioly MA, Gharabaghi A, Liebsch M, Carvalho $\mathrm{CH}$, Aguiar PH, Tatagiba M: Quantitative parameters of facial motor evoked potential during vestibular schwannoma surgery predict postoperative facial nerve function. Acta Neurochir (Wien) 153:1169-1179, 2011

2. Acioly MA, Liebsch M, Carvalho $\mathrm{CH}$, Gharabaghi A, Tatagiba M: Transcranial electrocortical stimulation to monitor the facial nerve motor function during cerebellopontine angle surgery. Neurosurgery 66 (6 Suppl Operative):354-362, 2010

3. Acioly MA, Liebsch M, de Aguiar PH, Tatagiba M: Facial nerve monitoring during cerebellopontine angle and skull base tumor surgery: a systematic review from description to current success on function prediction. World Neurosurg [epub ahead of print], 2011

4. Akagami R, Dong CC, Westerberg BD: Localized transcranial electrical motor evoked potentials for monitoring cranial nerves in cranial base surgery. Neurosurgery 57 (1 Suppl): 78-85, 2005

5. Calancie B, Harris W, Brindle GF, Green BA, Landy HJ: Threshold-level repetitive transcranial electrical stimulation for intraoperative monitoring of central motor conduction. J Neurosurg 95 (2 Suppl):161-168, 2001

6. Calancie B, Molano MR: Alarm criteria for motor-evoked potentials: what's wrong with the "presence-or-absence" approach? Spine (Phila Pa 1976) 33:406-414, 2008

7. Dong CCJ, Macdonald DB, Akagami R, Westerberg B, Alkhani A, Kanaan I, et al: Intraoperative facial motor evoked potential monitoring with transcranial electrical stimulation during skull base surgery. Clin Neurophysiol 116:588-596, 2005

8. Fukuda M, Oishi M, Takao T, Saito A, Fujii Y: Facial nerve motor-evoked potential monitoring during skull base surgery predicts facial nerve outcome. J Neurol Neurosurg Psychiatry 79:1066-1070, 2008

9. Goto T, Muraoka H, Kodama K, Hara Y, Yako T, Hongo K: 


\section{FNMEPs analyzed using the threshold-level method}

Intraoperative monitoring of motor evoked potential for the facial nerve using a cranial peg-screw electrode and a "threshold-level" stimulation method. Skull Base 20:429-434, 2010

10. House JW, Brackmann DE: Facial nerve grading system. Otolaryngol Head Neck Surg 93:146-147, 1985

11. Lyon R, Feiner J, Lieberman JA: Progressive suppression of motor evoked potentials during general anesthesia: the phenomenon of "anesthetic fade." J Neurosurg Anesthesiol 17: 13-19, 2005

12. Matthies C, Raslan F, Schweitzer T, Hagen R, Roosen K, Reiners K: Facial motor evoked potentials in cerebellopontine angle surgery: technique, pitfalls and predictive value. Clin Neurol Neurosurg 113:872-879, 2011

13. Mendiratta A, Emerson RG: Transcranial electrical MEP with muscle recording, in Nuwer MR (ed): Intraoperative Monitoring of Neural Function. Amsterdam: Elsevier, 2008, Vol 8 , pp 260-272

14. Neuloh G, Bogucki J, Schramm J: Intraoperative preservation of corticospinal function in the brainstem. J Neurol Neurosurg Psychiatry 80:417-422, 2009

15. Neuloh G, Pechstein U, Cedzich C, Schramm J: Motor evoked potential monitoring with supratentorial surgery. Neurosurgery 54:1061-1072, 2004

16. Sarnthein J, Bozinov O, Melone AG, Bertalanffy H: Motorevoked potentials (MEP) during brainstem surgery to preserve corticospinal function. Acta Neurochir (Wien) 153: $1753-1759,2011$
17. Szelényi A, Hattingen E, Weidauer S, Seifert V, Ziemann U: Intraoperative motor evoked potential alteration in intracranial tumor surgery and its relation to signal alteration in postoperative magnetic resonance imaging. Neurosurgery 67: 302-313, 2010

18. Szelényi A, Kothbauer KF, Deletis V: Transcranial electric stimulation for intraoperative motor evoked potential monitoring: Stimulation parameters and electrode montages. Clin Neurophysiol 118:1586-1595, 2007

19. Szelényi A, Langer D, Kothbauer K, De Camargo AB, Flamm ES, Deletis V: Monitoring of muscle motor evoked potentials during cerebral aneurysm surgery: intraoperative changes and postoperative outcome. J Neurosurg 105:675-681, 2006

20. Ulkatan S, Deletis V, Fernandez-Conejero I: Central or peripheral activations of the facial nerve? J Neurosurg 106:519-520, 2007 (Letter)

Manuscript submitted November 15, 2012.

Accepted December 21, 2012.

Please include this information when citing this paper: DOI: 10.3171/2012.12.FOCUS12386.

Address correspondence to: Johannes Sarnthein, Ph.D., Klinik für Neurochirurgie, UniversitätsSpital Zürich, 8091 Zürich, Switzerland. email: johannes.sarnthein@usz.ch. 